\title{
On generalized sequence spaces via modulus function
}

Ekrem Savaş*

\section{*Correspondence:}

ekremsavas@yahoo.com

Department of Mathematics, Istanbul Commerce University,

Sütlüce, Istanbul, Turkey

\begin{abstract}
In this paper, we introduce and study the concept of lacunary strongly

$(\mathbf{A}, \varphi)$-convergence with respect to a modulus function and lacunary $(\mathbf{A}, \varphi)$-statistical convergence and examine some properties of these sequence spaces. We establish some connections between lacunary strongly $(\mathbf{A}, \varphi)$-convergence and lacunary

$(\mathbf{A}, \varphi)$-statistical convergence.
\end{abstract}

MSC: Primary 40H05; secondary 40C05

Keywords: modulus function; almost convergence; lacunary sequence; $\varphi$-function; statistical convergence

\section{Introduction}

Let $s$ denote the set of all real and complex sequences $x=\left(x_{k}\right)$. By $l_{\infty}$ and $c$, we denote the Banach spaces of bounded and convergent sequences $x=\left(x_{k}\right)$ normed by $\|x\|=\sup _{n}\left|x_{n}\right|$, respectively. A linear functional $L$ on $l_{\infty}$ is said to be a Banach limit [1] if it has the following properties:

(1) $L(x) \geq 0$ if $n \geq 0$ (i.e. $x_{n} \geq 0$ for all $n$ ),

(2) $L(e)=1$, where $e=(1,1, \ldots)$,

(3) $L(D x)=L(x)$, where the shift operator $D$ is defined by $D\left(x_{n}\right)=\left\{x_{n+1}\right\}$.

Let $B$ be the set of all Banach limits on $l_{\infty}$. A sequence $x \in \ell_{\infty}$ is said to be almost convergent if all Banach limits of $x$ coincide. Let $\hat{c}$ denote the space of almost convergent sequences. Lorentz [2] has shown that

$$
\hat{c}=\left\{x \in l_{\infty}: \lim _{m} t_{m, n}(x) \text { exists uniformly in } n\right\},
$$

where

$$
t_{m, n}(x)=\frac{x_{n}+x_{n+1}+x_{n+2}+\cdots+x_{n+m}}{m+1} .
$$

By a lacunary $\theta=\left(k_{r}\right) ; r=0,1,2, \ldots$, where $k_{0}=0$, we shall mean an increasing sequence of non-negative integers with $k_{r}-k_{r-1} \rightarrow \infty$ as $r \rightarrow \infty$. The intervals determined by $\theta$ will be denoted by $I_{r}=\left(k_{r-1}, k_{r}\right]$ and $h_{r}=k_{r}-k_{r-1}$. The ratio $\frac{k_{r}}{k_{r-1}}$ will be denoted by $q_{r}$. The space of lacunary strongly convergent sequences $N_{\theta}$ was defined by Freedman et al. [3] as follows:

$$
N_{\theta}=\left\{x=\left(x_{k}\right): \lim _{r} \frac{1}{h_{r}} \sum_{k \in I_{r}}\left|x_{k}-l\right|=0 \text {, for some } l\right\} .
$$

O2014 Savaş; licensee Springer. This is an Open Access article distributed under the terms of the Creative Commons Attribution License (http://creativecommons.org/licenses/by/2.0), which permits unrestricted use, distribution, and reproduction in any medium, provided the original work is properly cited. 
In the special case where $\theta=\left(2^{r}\right)$ (see [3]) we have $N_{\theta}=w$, which is defined by

$$
w=\left\{x=\left(x_{k}\right): \lim _{n} \frac{1}{n} \sum_{k=0}^{n}\left|x_{k}-l\right|=0 \text {, for some } l\right\} .
$$

Das and Mishra [4] have introduced the space $A C_{\theta}$ of lacunary almost convergent sequences and the space $\left|A C_{\theta}\right|$ of lacunary strongly almost convergent sequences as follows:

$$
A C_{\theta}=\left\{x=\left(x_{k}\right): \lim _{r} \frac{1}{h_{r}} \sum_{k \in I_{r}}\left(x_{k+n}-L\right)=0, \text { for some } L \text { uniformly in } n\right\}
$$

and

$$
\left|A C_{\theta}\right|=\left\{x=\left(x_{k}\right): \lim _{r} \frac{1}{h_{r}} \sum_{k \in I_{r}}\left|x_{k+n}-L\right|=0 \text {, for some } L \text { uniformly in } n\right\} .
$$

Ruckle used the idea of a modulus function $f$ to construct a class of $F K$ spaces,

$$
L(f)=\left\{x=\left(x_{k}\right): \sum_{k=1}^{\infty} f\left(\left|x_{k}\right|\right)<\infty\right\}
$$

The space $L(f)$ is closely related to the space $l_{1}$, which is an $L(f)$ space with $f(x)=x$ for all real $x \geq 0$.

In 1999, Savaş [5] generalized the concept of strong almost convergence by using a modulus $f$ and $p=\left(p_{k}\right)$ is a sequence of strictly positive real numbers as follows:

$$
[\hat{c}(f, p)]=\left\{x: \lim _{n} \frac{1}{n} \sum_{k=1}^{n} f\left(\left|x_{k+m}-L\right|\right)^{p_{k}}=0, \text { for some } L, \text { uniformly in } m\right\}
$$

and

$$
[\hat{c}(f, p)]_{0}=\left\{x: \lim _{n} \frac{1}{n} \sum_{k=1}^{n} f\left(\left|x_{k+m}\right|\right)^{p_{k}}=0 \text {, uniformly in } m\right\} .
$$

More investigations in this direction and more applications of the modulus can be found in $[6-12]$.

Following Ruckle [13], a modulus function $f$ is a function from $[0, \infty)$ to $[0, \infty)$ such that

(i) $f(x)=0$ if and only if $x=0$,

(ii) $f(x+y) \leq f(x)+f(x)$ for all $x, y \geq 0$,

(iii) $f$ increasing,

(iv) $f$ is continuous from the right at zero.

By a $\varphi$-function we understand a continuous non-decreasing function $\varphi(u)$ defined for $u \geq 0$ and such that $\varphi(0)=0, \varphi(u)>0$, for $u>0$ and $\varphi(u) \rightarrow \infty$ as $u \rightarrow \infty$.

A $\varphi$-function $\varphi$ is called non-weaker than a $\varphi$-function $\psi$ if there are constants $c, b, k, l>$ 0 such that $c \psi(l u) \leq b \varphi(k u)$ (for all large $u$ ) and we write $\psi \prec \varphi$.

A $\varphi$-function $\varphi$ and $\psi$ are called equivalent if there are positive constants $b_{1}, b_{2}, c, k_{1}$, $k_{2}, l$ such that $b_{1} \varphi\left(k_{1} u\right) \leq c \psi(l u) \leq b_{2} \varphi\left(k_{2} u\right)$ (for all large $u$ ) and we write $\varphi \sim \psi$. 
A $\varphi$-function $\varphi$ is said to satisfy the condition $\left(\Delta_{2}\right)$ (for all large $u$ ) if for some constant $k>1$ there is satisfied the inequality $\varphi(2 u) \leq k \varphi(u)$ (see [12, 14]).

In this paper, we introduce and study some properties of the following sequence space which is generalization of Savaş [14].

\section{Main results}

Let $\varphi$ and $f$ be a given $\varphi$-function and modulus function, respectively, and let $p=\left(p_{n}\right)$ be a sequence of positive real numbers. Moreover, let $\mathbf{A}=\left(\mathbf{A}_{i}\right)$ be the generalized three parametric real matrix with $A_{i}=\left(a_{n, k}(i)\right)$ and a lacunary sequence $\theta$ be given. Then we define the following sequence spaces:

$$
N_{\theta}^{0}(\mathbf{A}, \varphi, f, p)=\left\{x=\left(x_{k}\right): \lim _{r} \frac{1}{h_{r}} \sum_{n \in I_{r}} f\left(\left|\sum_{k=1}^{\infty} a_{n k}(i) \varphi\left(\left|x_{k}\right|\right)\right|\right)^{p_{n}}=0 \text {, uniformly in } i\right\} .
$$

If $x \in N_{\theta}^{0}(\mathbf{A}, \varphi, f)$, the sequence $x$ is said to be lacunary strong $(\mathbf{A}, \varphi)$-convergent to zero with respect to a modulus $f$. When $\varphi(x)=x$ for all $x$, we obtain

$$
N_{\theta}^{0}(\mathbf{A}, f, p)=\left\{x=\left(x_{k}\right): \lim _{r} \frac{1}{h_{r}} \sum_{n \in I_{r}} f\left(\left|\sum_{k=1}^{\infty} a_{n k}(i) x_{k}\right|\right)^{p_{n}}=0 \text {, uniformly in } i\right\} .
$$

If we take $f(x)=x$, we write

$$
N_{\theta}^{0}(\mathbf{A}, \varphi, p)=\left\{x=\left(x_{k}\right): \lim _{r} \frac{1}{h_{r}} \sum_{n \in I_{r}}\left|\sum_{k=1}^{\infty} a_{n k}(i) \varphi\left(\left|x_{k}\right|\right)\right|^{p_{n}}=0 \text {, uniformly in } i\right\} .
$$

If we take $p_{k}=p$, for all $k$, we have

$$
N_{\theta}^{0}(\mathbf{A}, \varphi, f)=\left\{x=\left(x_{k}\right): \lim _{r} \frac{1}{h_{r}} \sum_{n \in I_{r}} f\left(\left|\sum_{k=1}^{\infty} a_{n k}(i) \varphi\left(\left|x_{k}\right|\right)\right|\right)^{p}=0 \text {, uniformly in } i\right\} .
$$

If we take $\mathbf{A}=I$ and $\varphi(x)=x$, respectively, then we have

$$
N_{\theta}^{0}=\left\{x=\left(x_{k}\right): \lim _{r} \frac{1}{h_{r}} \sum_{k \in I_{r}} f\left(\left|x_{k}\right|\right)^{p_{n}}=0\right\} .
$$

If we define the matrix $A=\left(a_{n k}(i)\right)$ as follows:

$$
a_{n k}(i):= \begin{cases}\frac{1}{n}, & \text { if } n \geq k \\ 0, & \text { otherwise }\end{cases}
$$

then we have

$$
\begin{aligned}
& N_{\theta}^{0}(\mathbf{C}, \varphi, f, p)=\left\{x=\left(x_{k}\right): \lim _{r} \frac{1}{h_{r}} \sum_{n \in I_{r}} f\left(\left|\frac{1}{n} \sum_{k=1}^{n} \varphi\left(\left|x_{k}\right|\right)\right|\right)^{p_{n}}=0, \text { uniformly in } i\right\}, \\
& a_{n k}(i):= \begin{cases}\frac{1}{n}, & \text { if } i \leq k \leq i+n-1, \\
0, & \text { otherwise, }\end{cases}
\end{aligned}
$$


then we have

$$
N_{\theta}^{0}(\hat{c}, \varphi, f, p)=\left\{x=\left(x_{k}\right): \lim _{r} \frac{1}{h_{r}} \sum_{n \in I_{r}} f\left(\left|\frac{1}{n} \sum_{k=i}^{i+n} \varphi\left(\left|x_{k}\right|\right)\right|\right)^{p_{n}}=0 \text {, uniformly in } i\right\} .
$$

If $x \in N_{\theta}^{0}(\hat{c}, \varphi, f)$, the sequence $x$ is said to be almost lacunary strong $\varphi$-convergent to zero with respect to a modulus $f$. In the next theorem we establish inclusion relations between $w(A, \varphi, f, p)$ and $N_{\theta}^{0}(\mathbf{A}, \varphi, f, p)$. We now have the following.

Theorem 2.1 Let $f$ be any modulus function and let there be a $\varphi$-function $\varphi$ and a generalized three parametric real matrix $\mathbf{A}$; let $p=\left(p_{n}\right)$ be a sequence of positive real numbers and the sequence $\theta$ be given. If

$$
w(\mathbf{A}, \varphi, f, p)=\left\{x=\left(x_{k}\right): \lim _{m} \frac{1}{m} \sum_{n=1}^{m} f\left(\left|\sum_{k=1}^{\infty} a_{n k}(i) \varphi\left(\left|x_{k}\right|\right)\right|\right)^{p_{n}}=0, \text { uniformly in } i\right\},
$$

then the following relations are true:

(a) If $\liminf _{r} q_{r}>1$ then we have $w(A, \varphi, f, p) \subseteq N_{\theta}^{0}(\mathbf{A}, \varphi, f, p)$.

(b) If $\sup _{r} q_{r}<\infty$, then we have $N_{\theta}^{0}(\mathbf{A}, \varphi, f, p) \subseteq w(A, \varphi, f, p)$.

(c) $1<\liminf _{r} q_{r} \leq \limsup _{r} q_{r}<\infty$, then we have $N_{\theta}^{0}(\mathbf{A}, \varphi, f, p)=w(A, \varphi, f, p)$.

Proof (a) Let us suppose that $x \in w(A, \varphi, f, p)$. There exists $\delta>0$ such that $q_{r}>1+\delta$ for all $r \geq 1$ and we have $h_{r} / k_{r} \geq \delta /(1+\delta)$ for sufficiently large $r$. Then, for all $i$,

$$
\begin{aligned}
& \frac{1}{k_{r}} \sum_{n=1}^{k_{r}} f\left(\left|\sum_{k=1}^{\infty} a_{n k}(i) \varphi\left(\left|x_{k}\right|\right)\right|\right)^{p_{n}} \\
& \geq \frac{1}{k_{r}} \sum_{n \in I_{r}} f\left(\left|\sum_{k=1}^{\infty} a_{n k}(i) \varphi\left(\left|x_{k}\right|\right)\right|\right)^{p_{n}} \\
& \quad=\frac{h_{r}}{k_{r}} \frac{1}{h_{r}} \sum_{n \in I_{r}} f\left(\left|\sum_{k=1}^{\infty} a_{n k}(i) \varphi\left(\left|x_{k}\right|\right)\right|\right)^{p_{n}} \\
& \geq \frac{\delta}{1+\delta} \frac{1}{h_{r}} \sum_{n \in I_{r}} f\left(\left|\sum_{k=1}^{\infty} a_{n k} \varphi\left(\left|x_{k}\right|\right)\right|\right)^{p_{n}} .
\end{aligned}
$$

Hence, $x \in N_{\theta}^{0}(\mathbf{A}, \varphi, f, p)$.

(b) If $\lim \sup _{r} q_{r}<\infty$ then there exists $M>0$ such that $q_{r}<M$ for all $r \geq 1$. Let $x \in$ $N_{\theta}^{0}(\mathbf{A}, \varphi, f, p)$ and $\varepsilon$ is an arbitrary positive number, then there exists an index $j_{0}$ such that for every $j \geq j_{0}$ and all $i$,

$$
R_{j}=\frac{1}{h_{j}} \sum_{n \in I_{r}} f\left(\left|\sum_{k=1}^{\infty} a_{n k}(i) \varphi\left(\left|x_{k}\right|\right)\right|\right)^{p_{n}}<\varepsilon .
$$

Thus, we can also find $K>0$ such that $R_{j} \leq K$ for all $j=1,2, \ldots$. Now let $m$ be any integer with $k_{r-1} \leq m \leq k_{r}$, then we obtain, for all $i$,

$$
I=\frac{1}{m} \sum_{n=1}^{m} f\left(\left|\sum_{k=1}^{\infty} a_{n k}(i) \varphi\left(\left|x_{k}\right|\right)\right|\right)^{p_{n}} \leq \frac{1}{k_{r-1}} \sum_{n=1}^{k_{r}} f\left(\left|\sum_{k=1}^{\infty} a_{n k}(i) \varphi\left(\left|x_{k}\right|\right)\right|\right)^{p_{n}}=I_{1}+I_{2},
$$


where

$$
\begin{aligned}
& I_{1}=\frac{1}{k_{r-1}} \sum_{j=1}^{j_{0}} \sum_{n \in I_{j}} f\left(\left|\sum_{k=1}^{\infty} a_{n k}(i) \varphi\left(\left|x_{k}\right|\right)\right|\right)^{p_{n}}, \\
& I_{2}=\frac{1}{k_{r-1}} \sum_{j=j_{0}+1}^{m} \sum_{n \in I_{j}} f\left(\left|\sum_{k=1}^{\infty} a_{n k}(i) \varphi\left(\left|x_{k}\right|\right)\right|\right)^{p_{n}} .
\end{aligned}
$$

It is easy to see that

$$
\begin{aligned}
I_{1} & =\frac{1}{k_{r-1}} \sum_{j=1}^{j_{0}} \sum_{n \in I_{j}} f\left(\left|\sum_{k=1}^{\infty} a_{n k}(i) \varphi\left(\left|x_{k}\right|\right)\right|\right)^{p_{n}} \\
& =\frac{1}{k_{r-1}}\left(\sum_{n \in I_{1}} f\left(\left|\sum_{k=1}^{\infty} a_{n k}(i) \varphi\left(\left|x_{k}\right|\right)\right|\right)^{p_{n}}+\cdots+\sum_{n \in I_{j}} f\left(\left|\sum_{k=1}^{\infty} a_{n k}(i) \varphi\left(\left|x_{k}\right|\right)\right|\right)^{p_{n}}\right) \\
& \leq \frac{1}{k_{r-1}}\left(h_{1} R_{1}+\cdots+h_{j_{0}} R_{j_{0}}\right) \\
& \leq \frac{1}{k_{r-1}} j_{0} k_{j_{0}} \sup _{1 \leq i \leq j_{0}} R_{i} \\
& \leq \frac{j_{0} k_{j_{0}}}{k_{r-1}} K .
\end{aligned}
$$

Moreover, we have for all $i$

$$
\begin{aligned}
I_{2} & =\frac{1}{k_{r-1}} \sum_{j=j_{0}+1}^{m} \sum_{n \in I_{j}} f\left(\left|\sum_{k=1}^{\infty} a_{n k} \varphi\left(\left|x_{k}\right|\right)\right|\right)^{p_{n}} \\
& =\frac{1}{k_{r-1}} \sum_{j=j_{0}+1}^{m} \frac{1}{h_{j}} \sum_{n \in I_{j}} f\left(\left|\sum_{k=1}^{\infty} a_{n k} \varphi\left(\left|x_{k}\right|\right)\right|\right)^{p_{n}} h_{j} \\
& \leq \varepsilon \frac{1}{k_{r-1}} \sum_{j=j_{0}+1}^{m} h_{j} \\
& \leq \varepsilon \frac{k_{r}}{k_{r-1}} \\
& =\varepsilon q_{r}<\varepsilon \cdot M .
\end{aligned}
$$

Thus $I \leq \frac{j_{0} k_{j_{0}}}{k_{r-1}} K+\varepsilon \cdot M$. Finally, $x \in w(A, \psi, f, p)$.

The proof of (c) follows from (a) and (b). This completes the proof.

Theorem 2.2 Let $f, f_{1}$, be modulus functions. Then we have

$$
N_{\theta}^{0}\left(A, f_{1}, \varphi, p\right) \subset\left(A, \varphi, f \circ f_{1}, p\right) .
$$

Proof This can be proved by using techniques similar to those used in the theorem of Savaş [14].

Recently Savaş [14] defined (A, $\varphi)$-statistical convergence as follows. 
Let $\theta$ be a lacunary sequence, and let $\mathbf{A}=\left(a_{n k}(i)\right)$ be the generalized three parametric real matrix, the sequence $x=\left(x_{k}\right)$, the $\varphi$-function $\varphi(u)$ and a positive number $\varepsilon>0$ be given. We write, for all $i$,

$$
K_{\theta}^{r}(A, \varphi, \varepsilon)=\left\{n \in I_{r}: \sum_{k=1}^{\infty} a_{n k}(i) \varphi\left(\left|x_{k}\right|\right) \geq \varepsilon\right\} .
$$

The sequence $x$ is said to be $(\mathbf{A}, \varphi)$-statistically convergent to a number zero if for every $\varepsilon>0$

$$
\lim _{r} \frac{1}{h_{r}} \mu\left(K_{\theta}^{r}(A, \varphi, \varepsilon)\right)=0, \quad \text { uniformly in } i
$$

where $\mu\left(K_{\theta}^{r}(A, \varphi, \varepsilon)\right)$ denotes the number of elements belonging to $K_{\theta}^{r}(\mathbf{A}, \varphi, \varepsilon)$. We denote by $S_{\theta}^{0}(\mathbf{A}, \varphi)$, the set of sequences $x=\left(x_{k}\right)$ which are lacunary $(\mathbf{A}, \varphi)$-statistical convergent to zero and we write

$$
S_{\theta}^{0}(\mathbf{A}, \varphi)=\left\{x=\left(x_{k}\right): \lim _{r} \frac{1}{h_{r}} \mu\left(K_{\theta}^{r}(A, \varphi, \varepsilon)\right)=0 \text {, uniformly in } i\right\} .
$$

More investigations in this direction can be found in [15-20].

We now establish inclusion relations between $N_{\theta}^{0}(\mathbf{A}, \varphi, f, p)$ and $S_{\theta}^{0}(A, \varphi)$.

In the following theorem we assume that $0<h=\inf p_{n} \leq p_{n} \leq \sup p_{k} \leq H \leq \infty$.

Theorem 2.3 (a) If the matrix $A$ and the sequence $\theta$ and functions $f$ and $\varphi$ are given, then

$$
N_{\theta}^{0}(A, \varphi, f, p) \subset S_{\theta}^{0}(A, \varphi) .
$$

(b) If the $\varphi$-function $\varphi(u)$ and the matrix $A$ are given, and if the modulus function $f$ is bounded, then

$$
S_{\theta}^{0}(A, \varphi) \subset N_{\theta}^{0}(A, \varphi, f, p) .
$$

Proof (a) Let $f$ be a modulus function and let $\varepsilon$ be a positive numbers. We write the following inequalities, for all $i$,

$$
\begin{aligned}
& \frac{1}{h_{r}} \sum_{n \in I_{r}} f\left(\left|\sum_{k=1}^{\infty} a_{n k}(i) \varphi\left(\left|x_{k}\right|\right)\right|\right)^{p_{n}} \\
& \quad=\frac{1}{h_{r}} \sum_{n \in I_{r}^{1}} f\left(\left|\sum_{k=1}^{\infty} a_{n k}(i) \varphi\left(\left|x_{k}\right|\right)\right|\right)^{p_{n}} \\
& \quad \geq \frac{1}{h_{r}} \sum_{n \in I_{r}^{1}}[f(\varepsilon)]^{p_{n}} \\
& \quad \geq \frac{1}{h_{r}} \sum_{n \in I_{r}^{1}} \min \left([f(\varepsilon)]^{\inf p_{n}},[f(\varepsilon)]^{H}\right) \\
& \geq \frac{1}{h_{r}} \mu\left(K_{\theta}^{r}(A, \varphi, \varepsilon)\right) \min \left([f(\varepsilon)]^{\inf p_{n}},[f(\varepsilon)]^{H}\right),
\end{aligned}
$$


where

$$
I_{r}^{1}=\left\{n \in I_{r}: \sum_{k=1}^{\infty} a_{n k}(i) \varphi\left(\left|x_{k}\right|\right) \geq \varepsilon\right\} .
$$

Finally, if $x \in N_{\theta}^{0}(A, \varphi, f, p)$ then $x \in S_{\theta}^{0}(A, \varphi, f)$.

(b) Let us suppose that $x \in S_{\theta}^{0}(A, \varphi)$. If the modulus function $f$ is a bounded function, then there exists an integer $K$ such that $f(x)<K$ for $x \geq 0$. Let us take

$$
I_{r}^{2}=\left\{n \in I_{r}: \sum_{k=1}^{\infty} a_{n k}(i) \varphi\left(\left|x_{k}\right|\right)<\varepsilon\right\}
$$

Thus we have, for all $i$,

$$
\begin{aligned}
\frac{1}{h_{r}} \sum_{n \in I_{r}} f\left(\left|\sum_{k=1}^{\infty} a_{n k}(i) \varphi\left(\left|x_{k}\right|\right)\right|\right)^{p_{n}} \\
\leq \frac{1}{h_{r}} \sum_{n \in I_{r}^{1}} f\left(\left|\sum_{k=1}^{\infty} a_{n k}(i) \varphi\left(\left|x_{k}\right|\right)\right|\right)^{p_{n}} \\
\quad+\frac{1}{h_{r}} \sum_{n \in I_{r}^{2}} f\left(\left|\sum_{k=1}^{\infty} a_{n k}(i) \varphi\left(\left|x_{k}\right|\right)\right|\right)^{p_{n}} \\
\leq \frac{1}{h_{r}} \sum_{n \in I_{r}^{1}} \max \left(K^{h}, K^{H}\right)+\frac{1}{h_{r}} \sum_{n \in I_{r}^{2}}[f(\varepsilon)]^{p_{n}} \\
\leq \max \left(K^{h}, K^{H}\right) \frac{1}{h_{r}} \mu\left(K_{\theta}^{r}(A, \varphi, \varepsilon)\right)+\max \left([f(\varepsilon)]^{h},[f(\varepsilon)]^{H}\right) .
\end{aligned}
$$

Taking the limit as $\varepsilon \rightarrow 0$, we observe that $x \in N_{\theta}^{0}(A, \varphi, f, p)$.

This completes the proof.

\section{Competing interests}

The author declares that they have no competing interests.

\section{Acknowledgements}

I would like to express my gratitude to the referee of the paper for his useful comments and suggestions towards the quality improvement of the paper. This paper was presented during the '2nd International Eurasian Conference on Mathematical Sciences and Applications' held on Sarajevo, Bosnia and Herzegovina on 26th to 29th August 2013, and it was submitted for the conference proceedings.

Received: 25 September 2013 Accepted: 4 February 2014 Published: 04 Mar 2014

\section{References}

1. Banach, S: Theorie des Operations Lineaires. Panstwowe Wydawnictwo Naukove, Warszawa (1932)

2. Lorentz, GG: A contribution to the theory of divergent sequences. Acta Math. 80, 167-190 (1948)

3. Freedman, AR, Sember, JJ, Raphel, M: Some Cesaro-type summability spaces. Proc. Lond. Math. Soc. 37, 508-520 (1978)

4. Das, G, Mishra, SK: Banach limits and lacunary strong almost convergence. J. Orissa Math. Soc. 2(2), 61-70 (1983)

5. Savaş, E: On some generalized sequence spaces defined by a modulus. Indian J. Pure Appl. Math. 30(5), 459-464 (1999)

6. Connor, J: On strong matrix summability with respect to a modulus and statistical convergence. Can. Math. Bull. 32(2), 194-198 (1989)

7. Maddox, IJ: Sequence spaces defined by a modulus. Math. Proc. Camb. Philos. Soc. 100, 161-166 (1986)

8. Malkowsky, E, Savaş, E: Some $\lambda$-sequence spaces defined by a modulus. Arch. Math. 36, 219-228 (2000) 
9. Nuray, F, Savaş, E: Some new sequence spaces defined by a modulus function. Indian J. Pure Appl. Math. 24(11), 657-663 (1993)

10. Pehlivan, S, Fisher, B: Some sequence spaces defined by a modulus. Math. Slovaca 43(3), 275-280 (1995)

11. Pehlivan, S: Sequence space defined by a modulus function. Erc. Univ. J. Sci. 3, 875-880 (1989)

12. Waszak, A: On the strong convergence in sequence spaces. Fasc. Math. 33, 125-137 (2002)

13. Ruckle, WH: FK Spaces in which the sequence of coordinate vectors is bounded. Can. J. Math. 25, $973-978$ (1973)

14. Savaş, E: On some new sequence spaces defined by infinite matrix and modulus. Adv. Differ. Equ. 2013, 274 (2013). doi:10.1186/1687-1847-2013-274

15. Fast, H: Sur la convergence statistique. Colloq. Math. 2, 241-244 (1951)

16. Šalát, T: On statistically convergent sequences of real numbers. Math. Slovaca 30, 139-150 (1980)

17. Fridy, JA: On statistical convergence. Analysis 5, 301-313 (1985)

18. Fridy, JA, Orhan, C: Lacunary statistical convergence. Pac. J. Math. 160, 43-51 (1993)

19. Savaş, E: Strongly almost convergence and almost $\lambda$-statistical convergence. Hokkaido Math. J. 29, 63-68 (2000)

20. Schoenberg, IJ: The integrability of certain functions and related summability methods. Am. Math. Mon. 66, 361-375 (1959)

10.1186/1029-242X-2014-101

Cite this article as: Savaş: On generalized sequence spaces via modulus function. Journal of Inequalities and Applications 2014, 2014:101

\section{Submit your manuscript to a SpringerOpen ${ }^{\circ}$ journal and benefit from:}

- Convenient online submission

- Rigorous peer review

Immediate publication on acceptance

Open access: articles freely available online

High visibility within the field

- Retaining the copyright to your article 